\title{
Despite cardiac denervation, atrial natriuretic peptides possess a cardiac sympathoinhibitory effect after heart transplantation
}

\author{
Bernard Geny, MD, PhD, Anne Charloux, MD, PhD, Gabrielle Brandenberger, PhD, and Francois Piquard, PhD, \\ Strasbourg, France
}

耳 eart transplantation is an excellent therapeutic alternative in end-stage heart failure, allowing a good survival for about half of heart transplant recipients until 15 years after surgical intervention.

However, surgically induced cardiac denervation increases the resting heart rate in heart transplant recipients through a withdrawal of the vagal tone. Blunting the heart rate reserve explains in part the reduced exercise capacity observed after heart transplantation. Additionally, with survival appearing to be inversely related to the degree of sympathetic activation and in particular to the noradrenergic drive to the diseased myocardium, ${ }^{1}$ it might be very interesting to modulate peptides with cardiac sympathoinhibitory activity after heart transplantation.

Atrial natriuretic peptides (ANPs) might be a good candidate. Indeed, cardiac natriuretic peptides are important diagnostic and prognostic tools, and their therapeutic interest is growing because of their diuretic, natriuretic, and vasodilatory properties. Thus ANP infusion is useful in poorly responsive oliguria after cardiac surgery. ${ }^{2}$ Moreover, importantly, ANPs possess sympathoinhibitory properties that might not only prevent against diuretic-induced sympathetic stimulation but that also reduce the basal sympathetic outflow. ${ }^{3}$ To date, there are several data concerning the renal and endocrine effect of ANPs after heart transplantation, ${ }^{4}$ but an eventual cardiac sympathoinhibitory action has not been evaluated in heart transplant recipients, probably because it appeared unlikely in cardiac-denervated patients.

However, mechanisms located beyond cardiac nerves have recently been shown to modulate the heart rate control, ${ }^{5}$ and we therefore hypothesized that despite cardiac denervation, an increase in endogenous ANP levels might result in cardiac sympathoinhibition after heart transplantation.

\section{Methods and Results}

After assessing cardiac innervation in 14 matched control subjects and heart transplant recipients, we investigated whether the neutral endopeptidase inhibition-induced increase in ANP levels might be associated with a reduced heart rate after heart transplantation during a 4-hour, double-blind, randomized, placebo-controlled study.

From Service de Physiologie et d'Explorations Fonctionnelles, Hôpitaux Universitaires et Institut de Physiologie, Faculté de Médecine, Strasbourg, France.

Received for publication Jan 3, 2006; accepted for publication Jan 24, 2006.

Address for reprints: Bernard P. Geny, MD, PhD, 11 rur Humann, Strasbourg 67000, France (E-mail: Bernard.Geny@ physio-ulp.u-strasbg.fr).

J Thorac Cardiovasc Surg 2006;131:1417-8

$0022-5223 / \$ 32.00$

Copyright $\odot 2006$ by The American Association for Thoracic Surgery doi:10.1016/j.jtcvs.2006.01.028

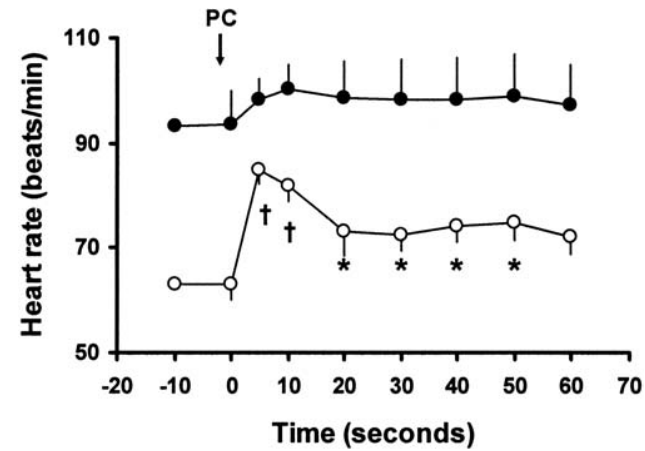

Figure 1. Heart rate response in controls () and heart-transplanted patients $(\mathrm{Htx})$ to an acute position change (PC) from prone to standing. Differences between groups, $P<0.01$ at all times. Difference with baseline, ${ }^{*} P<0.05,{ }^{\dagger} P<0.01$.

As expected, resting heart rate $(81.7 \pm 3.8$ vs $68.3 \pm 3.1$ beats/min, $P<.02)$, systemic blood pressure $(137 \pm 5$ vs $115 \pm$ $4 \mathrm{~mm} \mathrm{Hg}, P<.01)$, and ANP level $(19.4 \pm 3.6$ vs $9.4 \pm 1.0$ $\mathrm{pmol} / \mathrm{L}, P<.02$ ) were increased in heart transplant recipients compared with values in control subjects. In opposition to data obtained in control subjects, heart rate failed to change significantly in response to the positional change in heart transplant recipients (Figure 1).

Unlike after placebo, neutral endopeptidase inhibition (ecadotril, $200 \mathrm{mg}$; Bioprojet, Paris, France) increased circulating ANP levels $(+40 \% \pm 7 \%, P<.01)$, and its second messenger, cyclic guanosine monophosphate $(+209 \% \pm 72 \%, P<.03)$. It is noteworthy that systemic blood pressure did not increase but heart rate was significantly reduced concomitantly with the ANP increase after neutral endopeptidase inhibition (Figure 2).

\section{Discussion}

This study shows for the first time that despite cardiac denervation, endogenous ANPs possess a cardiac sympathoinhibitory effect after heart transplantation.

Moving from the lying to the standing position is an accepted noninvasive method to investigate sinus node innervation. Thus in innervated control subjects the vagal tone is immediately withdrawn so that the sinus node is no longer functionally inhibited by the parasympathetic activity. It results in a sharp and transient increase in heart rate. The lack of significant heart rate change in heart transplant recipients demonstrates their cardiac denervation. Heart rate decrease after ecadotril is likely to be related to ANP level because in the placebo arm of the study, the lack of change in ANP level was associated with a lack of heart rate change. 


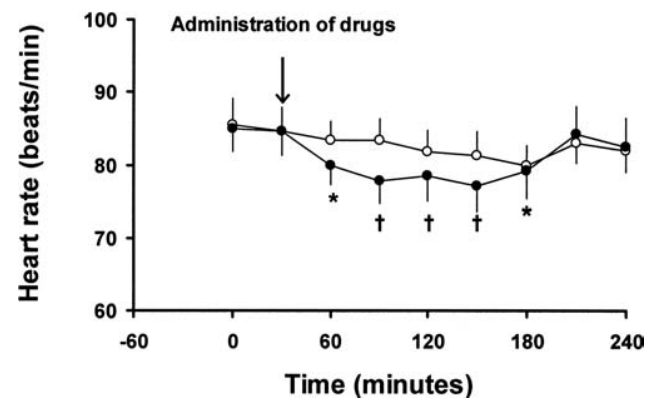

Figure 2. Time course of heart rate in Htx after administration of placebo () or neutral-endopeptidase inhibition (). Difference with baseline, ${ }^{*} P<0.05,{ }^{\dagger} P<0.01$.

Concerning the pathways involved, ANPs could decrease the sympathetic nerve traffic indirectly as a consequence of their hemodynamic action, through neural mechanisms, or both. However, the nonhypotensive stimulus required to demonstrate an inhibitory effect of ANPs on cardiac sympathetic outflow has been obtained in this study, and thus the heart rate response was unlikely to be related to modification in arterial baroreceptor loading. ${ }^{3}$

Thus a direct cardiac sympathoinhibitory effect of ANPs deserves to be discussed. In cardiac denervated patients only mechanisms located beyond cardiac nerves, such as reduction in sympathetic ganglionic neurotransmission or reduced effect of circulating catecholamines on the sinus node, might be involved. This information cannot be inferred from our data. However, an efferent mechanism, withdrawal of the inhibitory effects of excess cardiac norepinephrine release on vagal modulation of sinoatrial discharge, has been recently observed in patients with heart failure during $\beta$-blockade. This mechanism might also apply after heart transplantation. ${ }^{5}$

In summary, despite cardiac denervation, increased endogenous ANP levels have a cardiac sympathoinhibitory effect after heart transplantation. This opens interesting therapeutic perspectives because such an effect might be beneficial both in patient survival rate and quality of life, and these results further support the potential interest of increasing cardiac hormone levels after heart transplantation.

\section{References}

1. Kaye DM, Lefkovits J, Jennings GL, Bergin P, Broughton A, Esler MD Adverse consequences of high sympathetic nervous activity in the failing human heart. J Am Coll Cardiol. 1995;26:1257-63.

2. Hayashi Y, Ohtani M, Hiraishi T, Kobayashi Y. Postoperative synthetic human atrial natriuretic peptide infusion and oral spironolactone administration for a patient with giant atria and low plasma level of atrial natriuretic peptide. J Thorac Cardiovasc Surg. 2002;124:206-9.

3. Luchner A, Schunkert H. Interactions between the sympathetic nervous system and the cardiac natriuretic peptide system. Cardiovasc Res. 2004;63:443-9.

4. Piquard F, Richard R, Charloux A, Doutreleau S, Hannedouche T, Brandenberger G, et al. Hormonal, renal, hemodynamic responses to acute neutral endopeptidase inhibition in heart transplant patients. J Appl Physiol. 2002;93:569-75.

5. Kubo T, Parker JD, Azevedo ER, Atchison DJ, Newton GE, Picton P, et al. Vagal heart rate responses to chronic beta-blockade in human heart failure relate to cardiac norepinephrine spillover. Eur J Heart Fail. 2005;7:878-81. 